\title{
Ulcerated gastric leiomyoma causing massive upper gastrointestinal bleeding: A case report
}

\author{
DARYL RAMAI $^{1,2}$, QIUXUE T. TAN ${ }^{2}$, SOFIA NIGAR $^{1}$, EMMANUEL OFORI $^{1}$, \\ DENZIL ETIENNE $^{1}$ and MADHAVI REDDY ${ }^{1}$
}

\begin{abstract}
${ }^{1}$ Division of Gastroenterology and Hepatology, The Brooklyn Hospital Center, Academic Affiliate of The Icahn School of Medicine at Mount Sinai, Clinical Affiliate of The Mount Sinai Hospital, Brooklyn, New York, NY 11201, USA;

${ }^{2}$ Department of Anatomical Sciences, St. George's University School of Medicine, True Blue, Grenada, West Indies
\end{abstract}

Received November 13, 2017; Accepted March 7, 2018

DOI: $10.3892 /$ mco.2018.1597

\begin{abstract}
Leiomyomas are rare, benign submucosal tumors originating from smooth muscle cells. The clinical presentation is usually asymptomatic, with excellent prognosis. We herein report the case of a 68-year-old female with a solitary leiomyoma found during esophagogastroduodenoscopy for hematemesis. Histopathological examination revealed smooth muscle proliferation supported by positive staining for smooth muscle myosin heavy chain and negative staining for CD117 and S-100, consistent with the marker expression pattern of leiomyoma. We report on the clinical presentation of this case, and discuss the origin, epidemiology, treatment and management of leiomyomas.
\end{abstract}

\section{Introduction}

Extrauterine manifestations of leiomyomas are rare. Within the gastrointestinal tract, leiomyomas predominately occur in the esophagus (1). Gastric leiomyomas are benign submucosal tumors composed of smooth muscle cells, and account for $2.5 \%$ of all gastric neoplasms $(2,3)$. Leiomyomas are most frequently encountered in patients aged 50-70 years and bear no gender predilection (4). These tumors are slow-growing and usually asymptomatic; however, they may become clinically evident when the overlying gastric mucosa ulcerates with ensuing hemorrhage. Herein, we describe the case of a 68-year-old female patient presenting with hematemesis and found to have a gastric submucosal lesion on esophagogastroduodenoscopy (EGD), which was diagnosed as a gastric leiomyoma following histopathological examination.

Correspondence to: Dr Daryl Ramai, Division of Gastroenterology and Hepatology, The Brooklyn Hospital Center, Academic Affiliate of The Icahn School of Medicine at Mount Sinai, Clinical Affiliate of The Mount Sinai Hospital, 121 Dekalb Avenue, Brooklyn, New York, NY 11201, USA

E-mail: dramai@sgu.edu

Key words: leiomyoma, benign gastric tumor, upper gastrointestinal bleeding, hematemesis, esophagogastroduodenoscopy

\section{Case report}

A 68-year-old female patient with a medical history of chronic gastritis, hypertension, hyperlipidemia, atrial fibrillation (not on anticoagulation), a cerebral vascular event with residual left-sided weakness, seizure disorder, iron deficiency anemia, and anemia of chronic disease, presented with a complaint of hematemesis. On physical examination, the abdomen was soft, non-tender and non-distended. The heart rate was 85 beats/min, the blood pressure was $123 / 80 \mathrm{mmHg}$, the respiratory rate was 20 breaths/min, with an oxygen saturation of $95 \%$ on room air and a temperature of $97.6^{\circ} \mathrm{F}$. The laboratory findings were as follows: Sodium $143 \mathrm{mmol} / 1$, potassium $3.6 \mathrm{mmol} / \mathrm{l}$, albumin $2.7 \mathrm{~g} / \mathrm{dl}$, alkaline phosphatase $47 \mathrm{U} / 1$, aspartate aminotransferase $13 \mathrm{U} / 1$, alanine aminotransferase $10 \mathrm{U} / 1$, hemoglobin $5.1 \mathrm{~g} / \mathrm{dl}$, hematocrit $17 \%$, white blood cell count $16.1 \mathrm{~K} / \mathrm{cmm}$, and platelet count $262 \mathrm{~K} / \mathrm{cmm}$.

An abdominal computed tomography (CT) scan revealed a mass lesion seen protruding from the gastric lesser curvature into the lumen, measuring $\sim 4.1 \times 2.6 \mathrm{~cm}$ (Fig. 1). There was no evidence of extension of the mass outside the walls of the stomach. During esophagogastroduodenoscopy (EGD), a 4-cm submucosal lesion was identified along the lesser curvature of the stomach near the cardia (Fig. 2). Two umbilicated areas of ulceration were seen over the lesion, with no active bleeding. The lesion was biopsied without excision. To histologically process the biopsied specimen, a section $\leq 3-\mathrm{mm}$ thick was used. The section was fixed using $10 \%$ neutral buffered formalin. Next, the section slide was left in a $65^{\circ} \mathrm{C}$ oven to melt the paraffin. Finally, staining protocol for hematoxylin and eosin staining were performed at room temperature for $45 \mathrm{~min}$. Following histopathological processing, light microscopy revealed that the lesion was composed of bundles of elongated cells with oblong nuclei, and a submucosa with smooth muscle cell proliferation. On immunohistochemical examination, the cells of the lesion were positive for smooth muscle myosin heavy chain and negative for CD117 (c-kit) and S-100; the proliferation index (Ki-67) was <10\% (Fig. 3). These findings were consistent with the diagnosis of gastric leiomyoma. Following the procedure, the same day, the patient made a good recovery. Consent was obtained from the patient 
regarding the publication of the case details and associated images.

\section{Discussion}

Leiomyomas of the gastric cardia appear as homogeneous, low-attenuation masses with an endoluminal growth pattern, often ranging from 1.3 to $4.7 \mathrm{~cm}$ in diameter $(5,6)$. These tumors may also be found in the corpus and antrum of the stomach. When the tumors grow to $>2 \mathrm{~cm}$, they are more likely to present with central ulceration (6). The differential diagnosis of leiomyomas includes gastrointestinal stromal tumors (GISTs) and schwannomas. Histologically, they appear as round, solitary lesions arising from the muscularis mucosae, muscularis propriae, and possibly from smooth muscle of the vessel wall in the bowel (3). Their benign nature is evidenced by the microscopic appearance of abundant hyperplastic smooth muscle cells with minimal mitotic activity and low c-kit expression (7). However, the pathogenesis of gastric leiomyomas remains largely unclear.

In the past, leiomyomas and GISTs were referred to interchangeably. However, it is clinically important to distinguish these two entities, as leiomyomas are benign, while GISTs may display malignant potential (8). True leiomyomas are strongly and diffusely positive for desmin and smooth muscle actin (7). Additionally, true leiomyomas and schwannomas stain negative for CD117 and CD34, while GISTs are positive for these markers $(9,10)$. Leiomyomas tend to occur in younger patients compared with GISTs (7). Leiomyosarcomas are far less common and can be differentiated from leiomyomas by their very high mitotic rate (11).

The clinical presentation of leiomyomas depends on their size and location. The majority of gastric leiomyomas are slow-growing and asymptomatic; therefore, they are usually found incidentally on EGD, surgical exploration, or at autopsy (12). When symptomatic, leiomyomas manifest with upper gastrointestinal bleeding, atypical epigastric pain or non-specific dyspepsia, generally due to mucosal ulceration (13). The predisposing factors associated with gastric leiomyoma bleeding include treatment with anticoagulants, non-steroidal anti-inflammatory drugs and

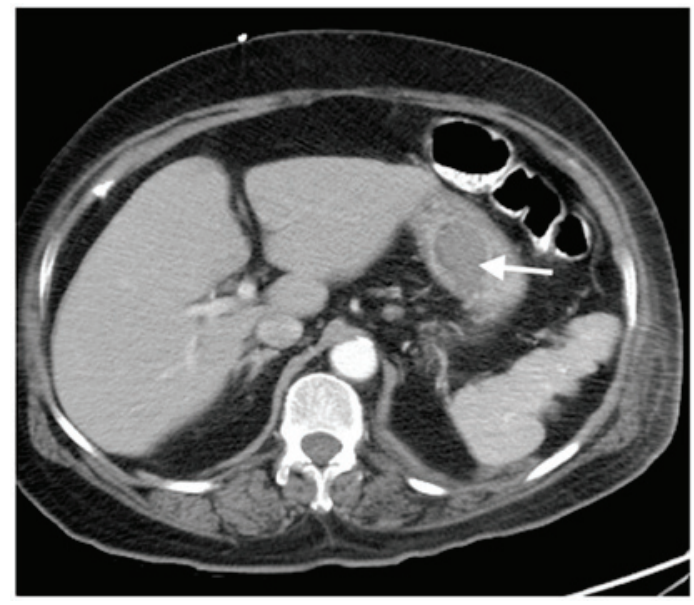

Figure 1. Computed tomography scan showing a gastric leiomyoma (arrow) protruding from the lesser curvature into the gastric lumen, measuring $\sim 4.1 \times 2.6 \mathrm{~cm}$.

corticosteroids (14-16). Endoscopically, leiomyomas appear as smooth, well-defined tumors, with stretched and effaced mucosal folds overlying the lesions, also referred to as the Schindler's sign (17).

According to the American Gastrointestinal Association (AGA), patients with submucosal tumors $<3 \mathrm{~cm}$ may be followed up by periodic EGD or endoscopic ultrasound examinations, while lesions $>3 \mathrm{~cm}$, in which the malignant potential cannot be determined by less invasive means, require surgical or endoscopic excision for diagnosis $(18,19)$. If the tumor is not removed, it may invade surrounding tissue. In conclusion, gastric leiomyoma is a rare, benign submucosal tumor originating from smooth muscle cells, most commonly found in the gastric cardia. It bears no predilection for gender and is most frequently found in patients aged 50-70 years. It is important to differentiate leiomyoma from leiomyosarcoma, which is a malignant tumor, and from GISTs, which possess malignant potential. Furthermore, according to AGA practice guidelines, surgical or endoscopic resection is recommended for tumors sized $>3 \mathrm{~cm}$. The patients with surgically resected tumors have a favorable clinical outcome.
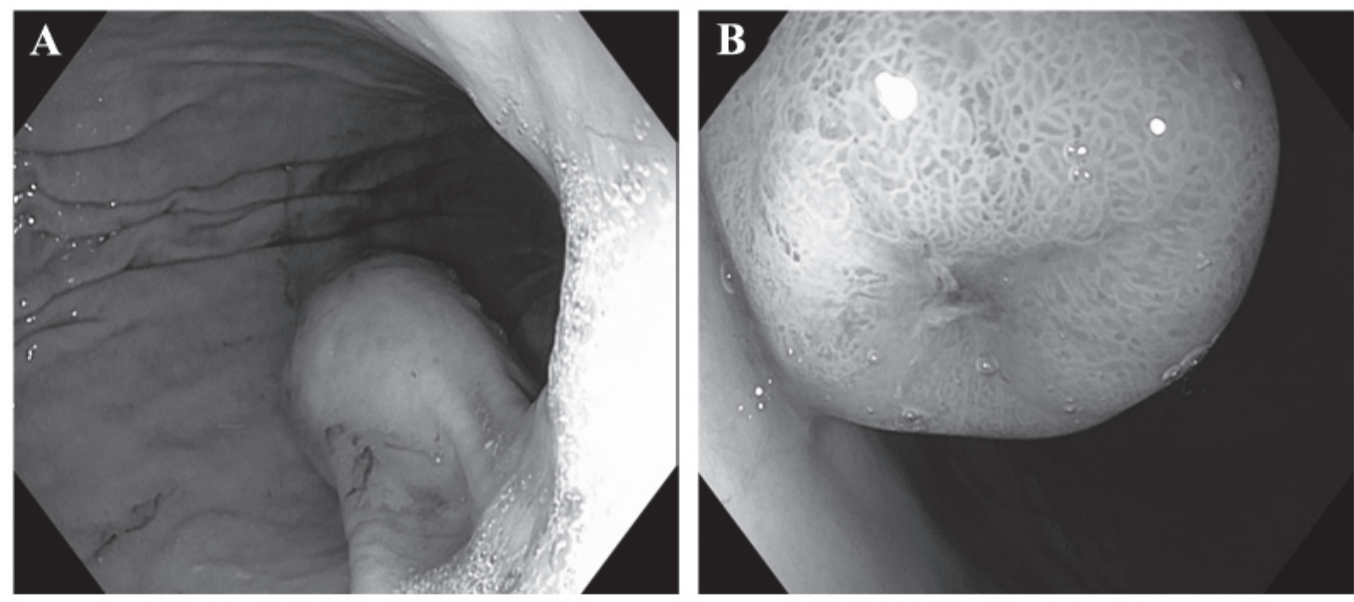

Figure 2. (A) Gastric submucosal lesion (leiomyoma) identified during endoscopy. (B) Central umbilication of the gastric leiomyoma. 

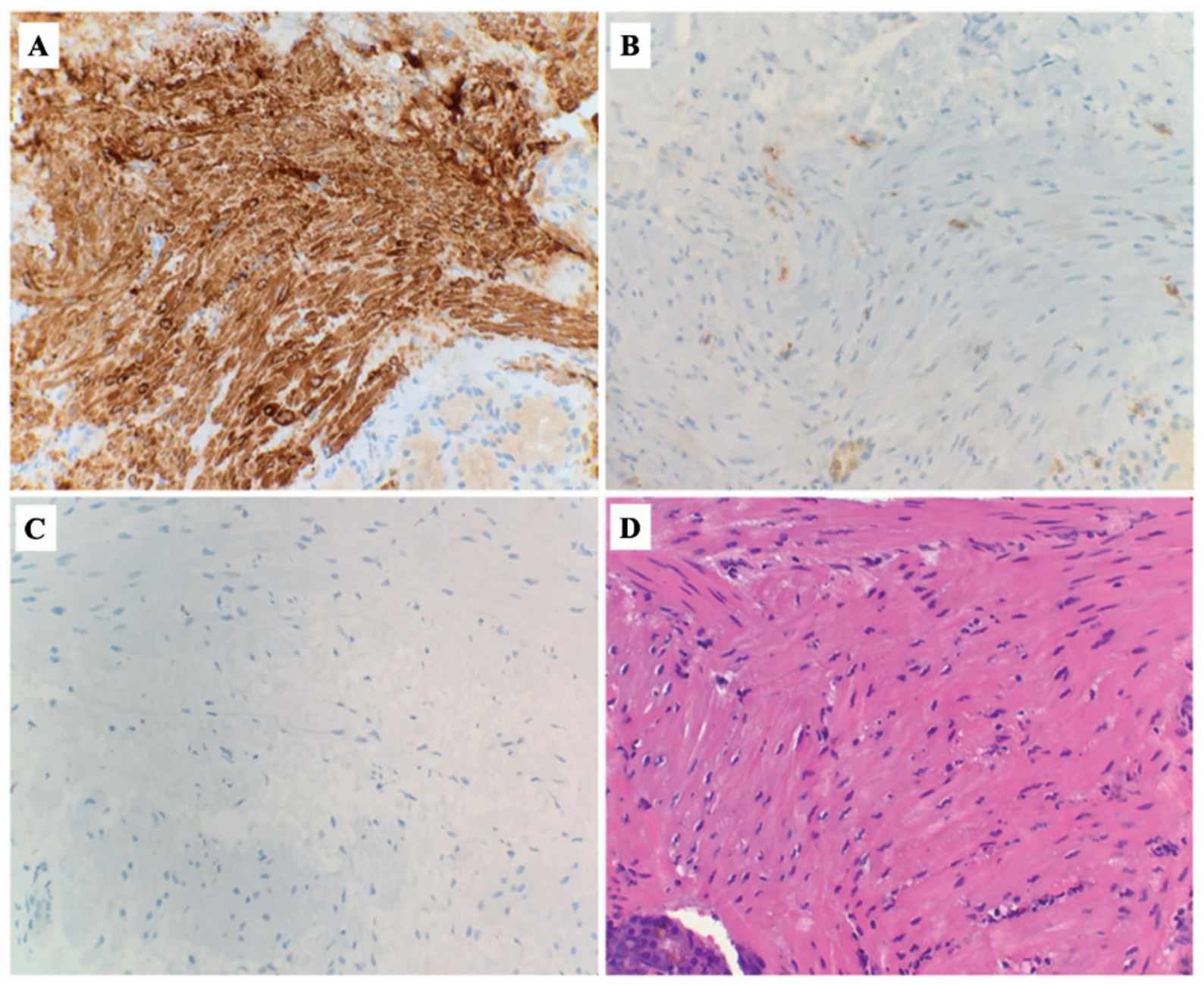

Figure 3. Cells of the lesion were (A) positive for smooth muscle myosin heavy chain and (B) negative for CD117. (C) Proliferation index (Ki-67) was low. (D) Lesion was composed of bundles of elongated cells with oblong nuclei (hematoxylin and eosin staining). All magnifications, $\mathrm{x} 40$.

\section{Acknowledgements}

Not applicable.

\section{Funding}

No funding was received.

\section{Authors' contributions}

DR, QTT and SN conceived and designed the study. DR and QTT drafted the manuscript. EO, SN, DE and MR critically revised the manuscript for intellectual content. All authors approved the manuscript for submission.

\section{Availability of data and materials}

Not applicable.

\section{Ethics approval}

This manuscript was acknowledged and approved by The Brooklyn Hospital Center Institutional Review Board on June 22, 2017 (no. 1082889-1).

\section{Consent for publication}

The patient provided written informed consent for the publication of the case details and associated images.

\section{Competing interests}

The authors declare that they have no competing interests.

\section{References}

1. Wang ZQ, Wang S, Ye YJ, Kang YL, Sun KK and Zheng HF: Gastrointestinal mesenchymal tumors: A clinical pathologic and immunohistochemical study of 210 cases. Zhonghua Wei Chang Wai Ke Za Zhi 10: 11-16, 2007 (In Chinese).

2. Morgan BK, Compton C, Talbert M, Gallagher WJ and Wood WC: Benign smooth muscle tumors of the gastrointestinal tract. A 24 year experience. Ann Surg 211: 63-66, 1990.

3. Agaimy A and Wünsch PH: True smooth muscle neoplasms of the gastrointestinal tract: Morphological spectrum and classification in a series of 85 cases from a single institute. Langenbecks Arch Surg 392: 75-81, 2007.

4. Gupta AK, Berry M and Mitra DK: Ossified gastric leiomyoma in a child: A case report. Pediatr Radiol 25: 48-49, 1995.

5. Hur BY, Kim SH, Choi JY, Rha SE, Lee MW, Kim SY, Han JK and Choi BI: Gastroduodenal glomus tumors: Differentiation from other subepithelial lesions based on dynamic contrast-enhanced CT findings. AJR Am J Roentgenol 197: 1351-1359, 2011. 
6. Lee MJ, Lim JS, Kwon JE, Kim H, Hyung WJ, Park MS, Kim MJ and Kim KW: Gastric true leiomyoma: Computed tomographic findings and pathological correlation. J Comput Assist Tomogr 31: 204-208, 2007.

7. Miettinen M, Sobin LH and Sarlomo-Rikala M Immunohistochemical spectrum of GISTs at different sites and their differential diagnosis with a reference to CD117 (KIT). Mod Pathol 13: 1134-1142, 2000.

8. Kang HC, Menias CO, Gaballah AH, Shroff S, Taggart MW Garg N and Elsayes KM: Beyond the GIST: Mesenchymal tumors of the stomach. Radiographics 33: 1673-1690, 2013.

9. Greenson JK: Gastrointestinal stromal tumors and other mesenchymal lesions of the gut. Mod Pathol 16: 366-375, 2003.

10. Ramai D, Lai J, Changela K, Reddy M and Shahzad G: Transverse colon schwannoma treated by endoscopic mucosal resection: A case report. Mol Clin Oncol 7: 830-832, 2017.

11. Miettinen M: Smooth muscle tumors of soft tissue and non-uterine viscera: Biology and prognosis. Mod Pathol 27 (Suppl 1): S17-S29, 2014.

12. Payne WG, Murphy CG and Grossbard LJ: Combined laparoscopic and endoscopic approach to resection of gastric leiomyoma. J Laparoendosc Surg 5: 119-122, 1995.

13. Matsuda M, Watanabe Y, Tonosu N, Nabeya Y, Arima H, Matsuzaki H, Ohira G, Sato H, Mizushima T and Uehara T: Hemoperitoneum secondary to exophytic leiomyoma: report of a case. Surg Today 30: 448-450, 2000.
14. Stalnikowicz R, Eliakim R, Ligumsky M and Rachmilewitz D: Drug-induced bleeding of gastric leiomyoma. Am J Gastroenterol 82: 419-420, 1987.

15. Saxton NL: Hemorrhage from a gastric leiomyoma during anticoagulant therapy. Report of a case. J Iowa State Med Soc 51: 717-721, 1961.

16. Din NA: Aspirin-induced haemorrhage in gastric leiomyoma. J R Coll Surg Edinb 18: 246-248, 1973.

17. Apostolopoulos P, Zalonis A, Karamoutzos A, Mavrogiannis P, Vlachou E, Tsibouris P and Alexandrakis G: Endoscopic submucosal dissection for the diagnosis and treatment of a gastric submucosal tumor: Initial experience in Greece. Ann Gastroenterol 25: 358-360, 2012.

18. Schindler R, Blomquist OA, et al: Leiomyosarcoma of the stomach; its roentgenologic and gastroscopic diagnosis and its possible relationship to pernicious anemia. Surg Gynecol Obstet 82: 239-252, 1946.

19. Hwang JH, Rulyak SD and Kimmey MB; American Gastroenterological Association Institute: American gastroenterological association institute technical review on the management of gastric subepithelial masses. Gastroenterology 130: 2217-2228, 2006. 